\title{
Integration of split ring resonators (SRRs) to UHF RFID tag antenna for size reduction
}

\begin{abstract}
This paper presents the design of miniaturized dipole antenna for passive Radio Frequency Identification (RFID). The proposed tag antenna is designed in such away that covers the Malaysian Ultra High - Frequency (UHF) RFID frequency band. The proposed tag antenna was designed using a double - sided Flame Retardant 4 (FR-4) substrate. The antenna's configuration consists of meander dipole antenna incorporated with metamaterial square split - ring resonators (SRRs) and capacitive tip - loading. This antenna designed focusing on the antenna parameters such as size, gain, radiation pattern, matching impedance and read range. In this work, the results show that the SRR structure is capable to reduce the size of the tag design by increasing the length of the unit cell.
\end{abstract}

Keyword: Radio frequency identification (RFID); Split ring resonators (SRRs); UHF RFID tag antenna; Doubleï sided flame retardant 4 (FR-4) 